Editorial

\title{
Are Heat Shock Proteins Druggable Candidates?
}

\author{
Tawanda Zininga and Addmore Shonhai \\ Department of Biochemistry, School of Mathematical and Natural Sciences, University of Venda, Thohoyandou, South Africa
}

Corresponding Author: Addmore Shonhai, Department of Biochemistry, School of Mathematical and Natural Sciences, University of Venda, Thohoyandou, South Africa. Email: adshon@gmail.com/addmore.shonhai@univen.ac.za

Heat shock proteins (Hsps) are ubiquitous and conserved molecules whose main function is to facilitate protein folding (molecular chaperone function). Some Hsps such as Hsp70 are also involved in protein folding, protein trafficking and ubiquitination of misfolded protein to facilitate their degradation (Bukau and Horwich, 1998). Hsps occur in functional networks and hence their coordinated expression is important in proteostasis. Some Hsps are stress-induced while others are expressed constitutively. Because of their role as sentinels of protein folding, it is not surprising that some Hsps are essential and for this reason, they constitute potential drug targets (Whitesell and Lindquist, 2005). However, their high conservation is a snag to their prospects as drug targets. In spite of this, drugs targeting some Hsps especially Hsp90 show great potential and some of them have entered clinical trials for cancer treatment (Jhaveri et al., 2012). In addition, there are prospects to target Hsps in treatment of infectious diseases such as malaria (reviewed in Shonhai, 2010; 2014).

Several factors appear to make Hsps ideal as druggable targets. The function of some Hsps is regulated by nucleotides. For example, Hsp70 and Hsp90 which constitute the most prominent druggable Hsps are ATPases. For this reason some of the most notable inhibitors of Hsps are ATP mimicks and other compounds that inhibit their ATPase activity (Banumathy et al., 2003; reviewed in Shonhai, 2010). In addition, the basal ATPase activities of Hsp90 and Hsp70 from different organisms is known to vary (Matambo et al., 2004; Vali et al., 2010). The ATPase activity of Hsp90 is known to increase up to 10 fold in cancer cells compared to its activity in normal cells (Vali et al., 2010). The enhanced activity of Hsp90 is thought to increase its client protein-processing turnover rate. Since some of Hsp90's client proteins are involved in signal transduction, it is conceivable that they are implicated in the development of cancer.

Some motifs of Hsps are structurally divergent across species. For example, Hsp70s contain a fairly divergent C-terminal Peptide Binding Domain (PBD). Some proline-rich antibacterial peptides that target the PBD of
DnaK (bacterial Hsp70) were reported not bind to human Hsp70 (Otvos et al., 2000). Hsp70s also possess sequence divergence in motifs that physically interact with their nucleotide exchange factors and for this reason, Hsp70s exhibit variable nucleotide exchange rates (Brehmer et al., 2001). Therefore nucleotide exchange of Hsp70 represents a functional aspect that could potentially be targeted in drug design.

Hsps exist in functional networks and therefore by abrogating their interaction it is possible to interfere with their function. For example, it is estimated that Hsp90 associates with at least 300 possible interactors (da Silva and Ramos, 2012). Although Hsp70 may interact with a smaller number of co-chaperones (molecules that regulate the chaperone activity of Hsps) compared to Hsp90, the former also associates with several cofactors such as Hsp40s. Hsp40s serve as substrate scanners of Hsp70 (Rüdiger et al., 2001). Therefore their role is to deliver substrates to Hsp70 as well as modulate its basal ATPase activity (Cyr et al., 1994; Cheetham and Caplan, 1998). The distribution of co-chaperones varies between species and between humans and parasites. For example, Plasmodium falciparum (the main agent of malaria) and Trypansoma brucei (which causes sleeping sickness in humans) possess a much bigger complement of Hsp40s than humans (Njunge et al., 2013; Burger et al., 2014). Some compounds that selectively target the interaction of Hsp40 with Hsp70 in malaria parasites have been shown to exhibit limited cytoxicity to human cells (Cockburn et al., 2011). Based on genomic data, it has been proposed that not all of 10 common co-chaperones of Hsp90 occur in obligate human parasites, suggesting that the distribution of co-chaperones of Hsp90 varies across species (Johnson and Brown, 2009).

There is growing evidence that the minor sequence variation between Hsps from malaria parasites represent adequate structural variation to facilitate their selective inhibition with minimum effects on their human counterparts (Cockburn et al., 2011; Wang et al., 2014). The variable post-transitional modifications that Hsp homologues undergo in different organisms and in diseased-and non-diseased cells presents make them 
functionally unique. For example, the phosphorylation of Hsp90 is known to regulate its chaperone function and hence its role in cell signaling (Mollapour et al., 2010). In addition, the expression levels of Hsps also vary in cancer and normal cells (Ghosh et al., 2008).

One of the major obstacles in targeting Hsps in drug design is that inhibition of one isoform of the protein could lead to up-regulation of another to provide compensatory function. It has been shown that down-regulation of either inducible or constitutively expressed Hsp70 did not result in the arrest of cancer cell growth (Schlecht et al., 2013). The cancer cells were only challenged when both the constitutive and inducible forms of the proteins were down-regulated (Schlecht et al., 2013). In addition, inhibition of Hsp90 has been shown to induce Hsp70 and Hsp27 (reviewed by Franke et al., 2013). Therefore targeting the functional association of Hsp70 and Hsp90 might be a more effective intervention than inhibiting each of the two molecular chaperones. Hsp70-Hsp90 organising protein (Hop) also known as Sti1 (Nicolet and Craig, 1989) serves as a module that brings Hsp70 and Hsp90 in a functional complex. A compound that inhibits Hop-Hsp90 association was shown to be lethal to cancer cells (Pimienta et al., 2011). Plasmodium falciparum Hop (PfHop) is fairly divergent from its human equivalence and for this reason, it has been proposed as a possible antimalarial drug target (Gitau et al., 2012).

Although Hsps are conserved, there is a growing body of evidence suggesting that Hsps are potential drug targets in spite of their conservation. The variable functional features that Hsps exhibit in normal versus diseased cells present an opportunity for possible selective inhibition of their function in target tissues. In addition, although apparently small sequence variations exist between Hsp homologues from parasites in comparison to their human counterparts, the fact that Hsps from a particular species may possess unique chaperone and co-chaperone partners presents a bottleneck for their selective inhibition. Since the function of proteins depends on their proper folding, inhibition of Hsps in combination drug therapies offers a promising prospect (Pesce et al., 2010; Shonhai, 2010). For example, compounds that inhibit Hsp90 have been shown to reverse chloroquine resistance in malaria parasites (Shahinas et al., 2013).

\section{References}

Banumathy, G., V. Singh, S.R. Pavithra and U. Tatu, 2003. Heat shock protein 90 function is essential for Plasmodium falciparum growth in human erythrocytes. J. Biol. Chem., 278: 18336-18345. DOI: $10.1074 /$ jbc.M211309200
Brehmer, D., S. Rudiger, C.S. Gassler, D. Klostermeier and L. Packschies et al., 2001. Tuning of chaperone activity of $\mathrm{Hsp} 70$ proteins by modulation of nucleotide exchange. Nature Structural Molecular Biol., 8: 427-432. DOI: 10.1038/87588

Bukau, B. and A.L. Horwich, 1998. The Hsp70 and Hsp60 chaperone machines. Cell, 92: 351-366.DOI: 10.1016/S0092-8674(00)80928-9

Burger, A., M.H. Ludewig and A. Boshoff, 2014. Investigating the chaperone properties of a novel heat shock protein, Hsp70.c, from Trypanosoma brucei. J. Parasitol. Res. Article, 2014: 1-12. DOI: 10.1155/2014/172582

Cheetham, M.E. and A.J. Caplan, 1998. Structure, function and evolution of DnaJ: Conservation and adaptation of chaperone function. Cell Stress Chaperones, $\quad 3: \quad 28-36$. http://www.ncbi.nlm.nih.gov/pmc/articles/PMC312 945/pdf/i1355-8145-003-01-0028.pdf

Cockburn, I.L., E-R. Pesce, J.M. Pryzborski, M.T. Davies-Coleman and P.G.K. Clark et al., 2011. Screening for small molecule modulators of Hsp70 chaperone activity using protein aggregation suppression assays: Inhibition of the plasmodial chaperone PfHsp70-1. Biol. Chem., 392: 431-438. DOI: $10.1515 /$ BC. 2011.040

Cyr, D.M., T. Langerand and M.G. Douglas, 1994. DnaJ-like proteins: Molecular chaperones and specific regulators of Hsp70. Trends Biochem. Sci., 19: 176-181. DOI: 10.1016/0968-0004(94)90281-X

da Silva, V.C.H. and C.H.I. Ramos, 2012. The network interaction of the human cytosolic $90 \mathrm{kDa}$ heat shock protein Hsp90: A target for cancer therapeutics. J. Proteomics, 75: 2790-2802. DOI: 10.1016/j.jprot.2011.12.028

Franke, J., S. Eichner, C. Zeilinger and A. Kirschning, 2013. Targeting heat-shock-protein 90 (Hsp90) by natural products: Geldanamycin, a show case in cancer therapy. Natural Products Reports, 30: 12991323. DOI: $10.1039 / C 3 N P 70012 G$

Ghosh, J.C., T. Dohi, B.H. Kang and D.C. Altieri, 2008. Hsp60 regulation of tumor cell apoptosis. J. Biol. Chem., 283: 5188-5194. DOI: 10.1074/jbc.M705904200

Gitau, G.W., P. Mandal, G.L. Blatch, J.P. Przyborski and A. Shonhai, 2012. Characterisation of the Plasmodium falciparum Hsp70-Hsp90 organising protein (PfHop). Cell Stress Chaperones, 17: 191202. DOI: $10.1007 / \mathrm{s} 12192-011-0299-\mathrm{x}$

Jhaveri, K., T. Taldone, S. Modi and G. Chiosis, 2012. Advances in the clinical development of heat shock protein 90 (Hsp90) inhibitors in cancers. Biochimicaet Biophysica Acta Molecular Cell Res., 1823: 742-755. DOI: 10.1016/j.bbamcr.2011.10.008 
Johnson, J.L. and C. Brown, 2009. Plasticity of the Hsp90 chaperone machinein divergent eukaryotic organisms. Cell Stress Chaperones, 14: 83-94. DOI: 10.1007/s12192-008-0058-9

Matambo, T.S., O.O. Odunuga, A. Boshoff and G.L. Blatch, 2004. Overproduction, purification and characterization of the Plasmodium falciparum heat shock protein 70. Protein Express. Purificat., 33: 214-222. DOI: 10.1016/j.pep.2003.09.010

Mollapour, M., S. Tsutsumi, A.C. Donnelly, K. Beebe and M.J. Tokita et al., 2010. Swe1Wee1-dependent tyrosine phosphorylation of $\mathrm{Hsp} 90$ regulates distinct facets of chaperone function. Molecular Cell, 37: 333-343. DOI: 10.1016/j.molcel.2010.01.005

Nicolet, C.M. and E.A. Craig, 1989. Isolation and characterization of STI1, a stress-inducible gene from Saccharomyces cerevisiae. Molecular Cell Biol., 9: 3638-3646. DOI: 10.1128/MCB.9.9.3638

Njunge, J.M., M.H. Ludewig, A. Boshoff, E. Pesce and G.L. Blatch, 2013. Hsp70s and J proteins of plasmodium parasites infecting rodents and primates: Structure, function, clinical relevance and drug targets. Current Pharmaceutical Design, 19: 387-403. DOI: 10.2174/138161213804143734

Otvos, L., O. Insug, M.E. Rogers, P.J. Consolvo and B.A. Condie et al., 2000. Interaction between heat shock proteins and antimicrobial peptides. Biochemistry, 39: 14150-14159. DOI: 10.1021/bi0012843

Pesce, E.R., I.L. Cockburn, J.L. Goble, L.L. Stephens and G.L. Blatch, 2010. Malaria heat shock proteins: Drug targets that chaperone other drug targets. Infec. Disorders-Drug Targets, 10: 147-157. DOI: $10.2174 / 187152610791163417$

Pimienta, G., K.M. Herbert and L. Regan, 2011. A compound that inhibits the HOP-Hsp90 complex formation and has unique killing effects in breast cancer cell lines. Molecular Pharmaceut., 8: 22522261. DOI: $10.1021 / \mathrm{mp} 200346 y$
Rüdiger, S., J. Schneider-Mergener and B. Bukau, 2001. Its substrate specificity characterizes the DnaJ cochaperone as a scanning factor for the DnaK chaperone. EMBO J., 20: 1042-1050. DOI: 10.1093/emboj/20.5.1042

Schlecht, R., S.R. Scholz, H. Dahmen, A. Wegener and C. Sirrenberg et al., 2013. Functional analysis of Hsp70 inhibitors. PLoS ONE, 8: 10.1371. DOI: 10.1371/journal.pone.0078443

Shahinas, D., A. Folefoc, T. Taldone, G. Chiosis and I. Crandall et al., 2013. A purine analog synergizes with Chloroquine (CQ) by targeting Hsp90 (PfHsp90). PLoS One, 8: e75446- e75446. DOI: 10.1371/journal.pone.0075446

Shonhai, A., 2010. Plasmodial heat shock proteins: Targets for chemotherapy. FEMS Immunol. Med. Microbiol., 58: 61-74. DOI: 10.1111/j.1574695X.2009.00639.x

Shonhai, A., 2014. Role of Hsp70s in Development and Pathogenicity of Plasmodium Species In: Heat Shock Proteins of Malaria, Shonhai, A. and G.L. Blatch (Eds), Springer UK, ISBN-10: 978-94-0077438-4, pp: 47-70.

Vali, S., R. Pallavi, S. Kapoor and U. Tatu, 2010. Virtual prototyping study shows increased ATPase activity of Hsp90 to be the key determinant of cancer phenotype. Syst. Synthetic Biol., 4: 25-33. DOI: 10.1007/s11693-009-9046-3

Wang, T., W.H. Bisson, P. Maser, L. Scapozza and D. Picard, 2014. Differences in conformational dynamics between plasmodium falciparum and human Hsp90 Orthologues enable the structurebased discovery of pathogen-selective inhibitors. J. Med. Chem., 57: 2524-2535. DOI: $10.1021 / \mathrm{jm} 401801$

Whitesell, L. and S.L. Lindquist, 2005. HSP90 and the chaperoning of cancer. Nature Rev. Cancer, 5: 761772. DOI: $10.1038 / \mathrm{nrc} 1716$ 